\title{
Piecing together a consensus on a TDP43-related dementia syndrome
}

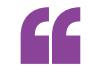

LATE has a public health impact that is right up there with $A D$

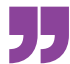

An international consensus group has provided the first comprehensive description of an amnestic dementia syndrome that is associated with TAR DNA-binding protein 43 (TDP43) proteinopathy. The condition, which the group has termed limbicpredominant age-related TDP43 encephalopathy (LATE), can be distinguished from other dementia subtypes on the basis of age of onset and distribution of TDP43 pathology in the brain.

TDP43 is a protein that normally resides in the cell nucleus and has a key role in the regulation of gene expression. TDP43 proteinopathy is characterized by cytoplasmic inclusions of phosphorylated TDP43, and is a prominent pathological feature in a number of neurodegenerative conditions, including frontotemporal lobar degeneration (FTLD) and amyotrophic lateral sclerosis.

The new report, which was published in Brain, pulls together multiple lines of evidence on LATE, including findings from clinical, pathological, neuroimaging and genetic studies. The authors provide guidance on the assessment and

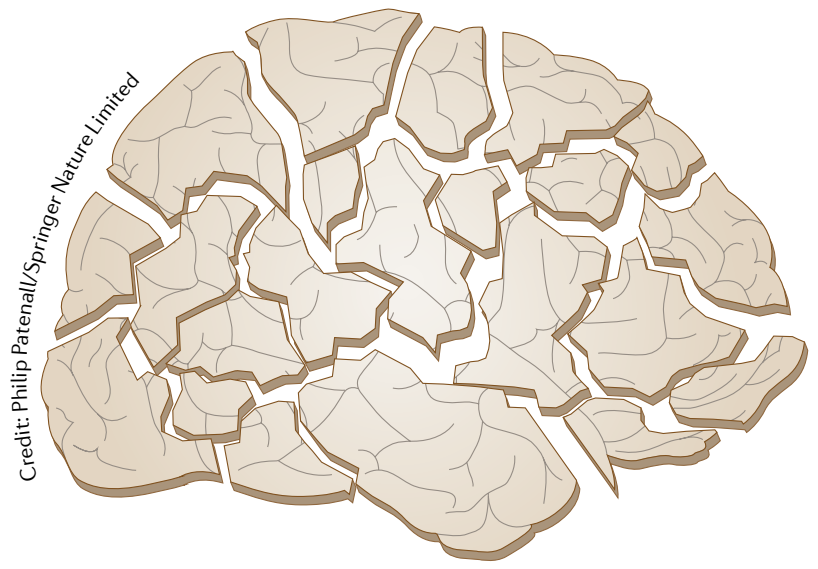

staging of LATE neuropathological change (LATE-NC) and identify avenues for future research.

"Researchers around the world have seen evidence of a common, impactful disease of ageing characterized by TDP 43 proteinopathy in the context of an Alzheimer disease (AD)-like clinical syndrome," explained corresponding author Peter Nelson. "For this consensus working group report, we analysed community-based cohort data to get an idea of the public health impact of this disease."

According to the new report, LATE typically affects people aged $\geq 80$ years. Although the clinical symptoms resemble $\mathrm{AD}$, the pathological findings place the condition closer to FTLD with TDP43 pathology (FTLD-TDP). However, the distribution of TDP43 proteinopathy in the brain is more restricted in LATE than in FTLD-TDP, with the amygdala, hippocampus and middle frontal gyrus being predominantly affected.

The authors suggest that the first description of LATE dates back to the 1990s. A study by Dennis Dickson and colleagues, which was published in 1994, showed that in individuals aged $\geq 80$ years, dementia and hippocampal sclerosis could occur in the absence of marked $\mathrm{AD}$ neuropathological change (ADNC). Subsequent research has shown that hippocampal sclerosis is a common but not invariable feature of LATE.

Large community-based autopsy studies indicate that LATE-NC is present in $>20 \%$ of people beyond the age of 80 years. "LATE is approximately 100 times more common than FTLD and affects people in an older age range than $\mathrm{AD}$," comments Nelson. "LATE has a public health impact that is right up there with $\mathrm{AD}$, but is massively underappreciated and understudied."

As Nelson and colleagues discuss in their report, the increasing recognition of LATE is likely to have important implications for the design of future clinical trials. In addition to providing a novel therapeutic target in its own right, LATE-NC will need to be taken into account in trials of drugs that target ADNC, as the two pathologies frequently coexist in the elderly population and can have distinct effects on cognitive performance.

The consensus group highlights a number of priorities for future investigation into LATE, including a search for biomarkers and further exploration of the underlying pathology and disease mechanisms. Genetic studies have already provided several leads, with risk variants for LATE being identified in the GRN, TMEM106B, ABCC9, $K C N M B 2$ and $A P O E$ genes.

"Animal models and basic science research into LATE are imperative, with the caveat that the aged human brain is challenging to model accurately," the authors conclude in their report. "Ultimately, it is hoped that these collective research efforts will one day result in successful preventive and therapeutic strategies."

Heather Wood

ORIGINAL ARTICLE Nelson, P. T. et al. Limbicpredominant age-related TDP-43 encephalopathy (LATE): consensus working group report. Brain https://doi.org/10.1093/brain/awz099 (2019) FURTHER READING Dickson, D. W. et al. Hippocampal sclerosis: a common pathological feature of dementia in very old ( $\geq 80$ years of age) humans. Acta Neuropathol. 88, 212-221 (1994)| Chen-Plotkin, A. S. et al. TAR DNA-binding protein 43 in neurodegenerative disease. Nat. Rev. Neurol. $6,211-220$ (2010) 